\title{
Insulinoma of Pancreatic Head Localized by Intra-Arterial Calcium Stimulation with Hepatic Venous Sampling \\ Siong Hu Wong ${ }^{1}$, Julia Omar ${ }^{2}$, Noor Azlin Azraini Che Soh Yusof ${ }^{3}$
}

\begin{abstract}
$\underline{\text { Abstract }}$
Hypoglycemia is a common medical emergency. Insulinoma, a rare pancreatic endocrine tumour is the most common cause of hypoglycemia in patients without systemic illness. Most insulinomas are small, solitary and benign, which can be treated effectively by surgical removal of the tumour. Diagnosis is based on clinical features, laboratory tests and radiological investigation to localize the tumour. However, small size of this tumour leads to difficulty in localization of the disease, especially in occult insulinoma. Intra-arterial calcium stimulation with hepatic venous sampling can be utilised for preoperative localization of the tumour. We hereby present a case of a 33-year-old woman presenting with history of recurrent hypoglycemic symptoms and morbid obesity. A thorough history, clinical examination and laboratory investigation including prolonged fasting test were conducted and diagnosis of insulinoma was made. Intra-arterial calcium stimulation with hepatic venous sampling was carried out and revealed localization of insulinoma at pancreatic head.
\end{abstract}

Kevwords: Insulinoma; preoperative localization; intra-arterial calcium stimulation with hepatic venous sampling

Bangladesh Journal of Medical Science Vol. 20 No. 01 January'21. Page : 216-219 DOI: https://doi.org/10.3329/bjms.v20i1.50376

\section{Introduction}

Pancreatic insulinoma is a relatively rare endocrine tumour with an estimated annual incidence of four in every one million persons ${ }^{1}$. It is female predominance, with mean age of presentation being fifth decade of life. Most insulinomas are solitary and small, with $90 \%$ being less than two $\mathrm{cm}^{2}$. Although $90 \%$ of these tumours are benign ${ }^{3}$, they have the potential to produce profound morbidity and risk of fatal hypoglycaemia. Insulinoma can be treated effectively by surgical excision. Hence, preoperative localization of the tumour is essential. Although a variety of non-invasive imaging modalities, such as transabdominal ultrasound (US), computed tomography (CT) and magnetic resonance imaging (MRI) have been highly favoured, preoperative localization fails $10-27 \%$ of the time, particularly in the case of occult insulinoma ${ }^{4}$. Occult insulinoma refers to a biochemically proven tumour with indeterminate anatomical site before operation. Intra-arterial calcium stimulation with hepatic venous sampling (ASVS), an invasive modality, is highly accurate in the preoperative localization of insulinomas and has been shown to be superior to non-invasive localization techniques ${ }^{3,4}$.

\section{Case report}

A 33-year-old woman was admitted to emergency

1. Siong $\mathrm{Hu}$ Wong

2. Julia Omar

3. Noor Azlin Azraini Che Soh Yusof Department of Chemical Pathology, School of Medical Sciences, Health Campus, Universiti Sains Malaysia, 16150 Kubang Kerian, Kelantan, Malaysia. and Hospital Universiti Sains Malaysia, Health Campus, USM, 16150, Kubang Kerian, Kelantan, Malaysia.

Correspondence to: Dr. Noor Azlin Azraini Che Soh@ Yusof, Department of Chemical Pathology, School of Medical Sciences, Health Campus, Universiti Sains Malaysia, 16150, Kubang Kerian, Kelantan, Malaysia. Email : noorazlin79@usm.my 
department after being found unconscious. She regained consciousness after administration of glucose. She gave history of episodic and repetitive hypoglycemic symptoms including light headedness, sweating, and palpitation for two years, and those symptoms resolved after taking snacks. She also noticed weight gain of around $20 \mathrm{~kg}$ in these two years. As she never had a major episode of loss of consciousness prior to current admission, she underestimated her condition and didn't seek for medical aid. She had no family history of endocrine disease. There were no prescription medications at the time of her evaluation. On physical examination, she measured $155 \mathrm{~cm}$ in height and weighed 107 $\mathrm{kg}$, giving a body mass index of $44.5 \mathrm{~kg} / \mathrm{m}^{2}$. Her vital signs were normal and abdominal examination showed no palpable mass or organomegaly.

Laboratory investigations during admission demonstrated a low plasma glucose level of 2.1 $\mathrm{mmol} / \mathrm{L}$, a high serum insulin level of $1444 \mathrm{pmol} / \mathrm{L}$ (reference range: $18-173 \mathrm{pmol} / \mathrm{L}$ ) and high serum C-peptide level of $3.80 \mathrm{nmol} / \mathrm{L}$ (reference range: $0.37-1.47 \mathrm{nmol} / \mathrm{L}$ ). Her serum cortisol level was normal suggesting intact pituitary adrenal axis. Insulin growth factor-1 and thyroid function tests were within normal range. 72-hour fasting test was then carried out and she produced symptomatic hypoglycemia with abnormally elevated insulin level of $168 \mathrm{pmol} / \mathrm{L}$ and C-peptide level of $1.5 \mathrm{nmol} / \mathrm{L}$. In view of the clinical picture and laboratory data, the clinical impression was that of an insulinoma.

Attempts to localize the insulinoma using noncontrast CT, contrast-enhanced CT, and MRI were unsuccessful. Hence, to localize the tumour, pancreatic angiography combined with ASVS was performed. $10 \%$ calcium gluconate solution was injected into four arteries in turns: proximal splenic artery (PSA), distal splenic artery (DSA), inferior duodenal pancreatic artery (IDPA) and superior duodenal pancreatic artery (SDPA). Hepatic venous blood was sampled for insulin and C-peptide at 0 second, 30 seconds, 60 seconds and 120 seconds after calcium injection. A twofold or higher step-up increase in insulin level in hepatic venous samples after the arterial calcium injection to a feeding artery was defined as positive response ${ }^{5}$. Although patient's pancreatic angiography showed no apparent blushing of pancreatic lesion, the venous sampling test showed positive over calcium injection into superior duodenal pancreatic artery (Figure 1). A diagnosis of insulinoma of pancreatic head was reached. Patient was counselled for resection of the insulinoma. However, patient was unwilling to undergo surgery, and she was given medical treatment including dietary modification and diazoxide.

\section{Discussion}

Hypoglycemia is a common medical emergency. Common causes include old age, inadequate food intake, overdose of medications (insulin or oral hypoglycemic agent), alcohol consumption, presence of concomitant infection, liver disease, chronic kidney disease and coronary artery disease ${ }^{6}$. Infrequently, hypoglycemia can also be caused by insulinoma. Insulinoma can be classified as sporadic or familial. Most insulinomas occur sporadically and are typically solitary, whereas familial insulinomas occur as multiple lesions, in conjunction with multiple endocrine neoplasia (MEN)-1 syndrome ${ }^{4}$.

Insulinoma secretes insulin intermittently, thereby causing episodic attack of hypoglycemia, which is

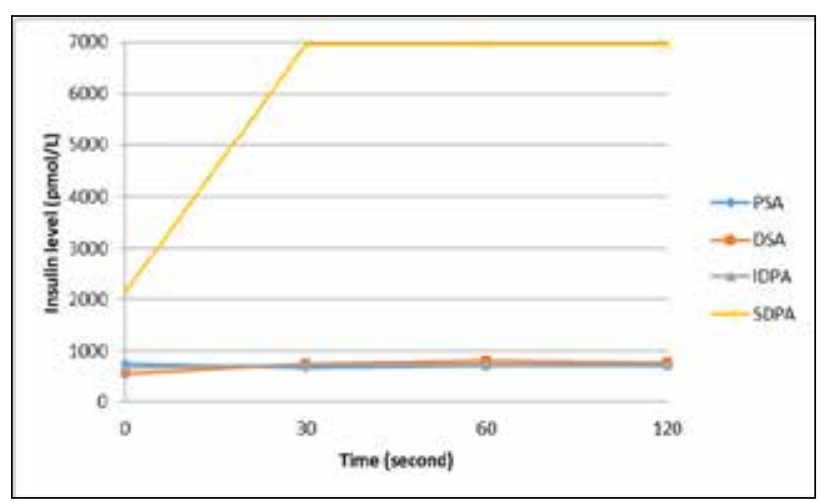

Figure 1. Hepatic venous insulin values obtained after injection of calcium gluconate into four arteries: PSA, DSA, IDPA, and SDPA. At least more than two-fold rise (value was more than upper detection limit of $6945 \mathrm{pmol} / \mathrm{L}$ ) in insulin level at $30 \mathrm{~s}$ in SDPA localized the tumour in the head of pancreas. PSA: Proximal splenic artery; DSA: Distal splenic artery; IDPA: Inferior duodenal pancreatic artery, SDPA: Superior duodenal pancreatic artery.

characterized by neuroglycopenic symptoms and sympathetic overdrive (catecholamine response) ${ }^{7}$. Common neuroglycopenic symptoms include confusion, visual changes, altered consciousness, amnesia, coma and convulsions, while common catecholamine response includes sweating, weakness, tremor, hunger, palpitation and feeling of warmth ${ }^{4,7}$. Obesity is also a frequent feature in patient with insulinoma as patient tends to increase frequency of food intake to avoid hypoglycemic symptoms ${ }^{4}$. 
Our patient experienced typical neuroglycopenic and sympathetic overdrive symptoms, and she also gained weight excessively within the two years of hypoglycemic episodes.

Diagnosing insulinoma accurately requires clear clinical observation and laboratory tests. Clinically, insulinoma is diagnosed by presence of Whipple's triad which consists of hypoglycemic symptoms provoked by fasting, plasma glucose level less than $50 \mathrm{mg} / \mathrm{dL}(2.8 \mathrm{mmol} / \mathrm{L})$ during the appearance of symptoms, and relief of symptoms after glucose administration $^{8}$. Measurement of plasma glucose, pro-insulin, insulin, and C-peptide during a 72hour fast has been gold standard for biochemical diagnosis of insulinoma for over 80 years $^{4,7}$. The rationale of this prolonged fasting test is to establish inappropriately high pro-insulin, insulin and C-peptide concentrations in the setting of low fasting plasma glucose concentration. According to Endocrine Society clinical practice guideline, the findings of hypoglycemic symptoms, signs, or both with fasting plasma glucose concentration $<55 \mathrm{mg}$ / $\mathrm{dL}(3.0 \mathrm{mmol} / \mathrm{L})$, pro-insulin $\geq 5.0 \mathrm{pmol} / \mathrm{L}$, insulin $\geq$ $3.0 \mu \mathrm{U} / \mathrm{mL}(18 \mathrm{pmol} / \mathrm{L})$, and C-peptide $\geq 0.6 \mathrm{ng} / \mathrm{mL}$ $(0.2 \mathrm{nmol} / \mathrm{L})$ establish endogenous hyperinsulinism ${ }^{9}$. Our patient displayed characteristics typical for insulinoma, including presence of Whipple's triad and endogenous hyperinsulinism established by $72-$ hour fasting test. Pro-insulin level was not measured as the test was not available in our hospital.

Once clinical and biochemical diagnosis of insulinoma is established, investigation to localize the tumour is carried out to aid management decision. Pre-operative localization aids in differentiation of solitary from multiple lesions and thus allows simple enucleation of a solitary tumour rather than unnecessary blind resection of the pancreas, attribute to higher operative success rate and reducing operative morbidity and mortality ${ }^{3}$. Non-invasive imaging modalities such as US, CT and MRI are highly preferred, but are often unsuccessful in insulinomas less than $2 \mathrm{~cm}$ in diameter. When non-invasive modalities fail to localize the tumours, invasive modalities such as endoscopic ultrasonography (EUS), angiography, transhepatic portal venous sampling, and ASVS are utilized. Comparing these two modalities, invasive ones have higher sensitivity compare to non-invasive ones, particularly ASVS technique which is reported to be the most sensitive technique with sensitivity of over $90 \%$ (range $87.5-100 \%$ ) in localization accuracy $^{2-4,7}$.

ASVS was introduced by Doppman et al. in 1991, whereby localization of pancreatic insulinoma is based on release of insulin from islet cell tumour after stimulation by secretagogue (calcium) $)^{5}$. The success of ASVS relies on few assumptions, including that the tumour has a dominant feeding artery, that calcium stimulates a characteristic release of insulin granules from tumour cells, and that the remain beta cells are suppressed from the chronic insulin hypersecretion ${ }^{10}$. However, it should be noted that this regionalization instead of exact localization, as the lesion is not visually identified precisely, but is identified according to its increased insulin secretion $^{3,9}$. An additional advantage of ASVS is to aid in distinguishing functional from non-functional tumour in MEN-1 patients with multiple lesions ${ }^{2}$. As in this case, attempts to localize patient's insulinoma with CT, MRI and pancreatic angiography were unsuccessful, but ASVS showed that the insulinoma was located at the pancreatic head region.

Insulinoma can be treated surgically or medically. Surgical excision is curative in most cases and hence is the treatment of choice ${ }^{4,7}$. Medical management generally is recommended to high-risk candidates for surgery, those unwilling for surgery, those who have undergone unsuccessful surgery with persistent symptoms and when surgery is technically impossible. It includes dietary modification and pharmacological treatment with diazoxide, calcium channel blockers and somatostatin analogues ${ }^{4,7}$.

Insulinoma is an uncommon neuroendocrine tumour, mostly benign, but can be life-threatening in causing fatal hypoglycemia. Biochemical diagnosis is obtained by 72-hour fast followed by imaging modalities to confirm the diagnosis and to localize the tumour. Preoperative localization remains challenging especially in the case of occult insulinoma as in this case. ASVS has been shown to be highly sensitive and accurate in localization of insulinoma which aid in patient's treatment planning.

Acknowledgements: None

Funding: None

Conflict of interest: None 
Insulinoma of Pancreatic Head Localized by Intra-Arterial Calcium Stimulation with Hepatic Venous Sampling

\section{References:}

1. Service FJ, McMahon MM, O’Brien PC, Ballard DJ. Functioning insulinoma-incidence, recurrence, and long-term survival of patients: a 60-year study. Mayo Clin Proc. 1991; 66:711-719.

2. Morganstein DL, Lewis DH, Jackson J, Isla A, Lynn J, Devendra D, Meeran K, Todd JF. The role of arterial stimulation and simultaneous venous sampling in addition to cross-sectional imaging for localisation of biochemically proven insulinoma. EurRadiol. 2009;19(10):2467-2473.

3. Sung YM, Do YS, Lee MK, Shin SW, Liu WC, Choo SW, Choo IW. Selective intra-arterial calcium stimulation with hepatic venous sampling for preoperative localization of insulinomas. Korean J Radiol. 2003;4(2):101-108.

4. Shin JJ, Gorden P, Libutti SK. Insulinoma: pathophysiology, localization and management. Future Oncol. 2010;6(2):229-237.

5. Doppman JL, Miller DL, Chang R, Shawker TH, Gorden P, Norton JA. Insulinomas: localization with selective intraarterial injection of calcium. Radiol. 1991
Jan;178(1):237-241.

6. Su YJ, Liao CJ. Hypoglycemia in Emergency Department. J Acute Dis. 2015;4(1):59-62.

7. Okabayashi T, Shima Y, Sumiyoshi T, Kozuki A, Ito S, Ogawa Y, Kobayashi M, Hanazaki K. Diagnosis and management of insulinoma. World $J$ Gastroenterol. 2013;19(6):829-837.

8. Whipple AO, Frantz VK. Adenoma of islet cells with hyperinsulinism: a review. Ann Surg. 1935;101(6):12991355.

9. Cryer PE, Axelrod L, Grossman AB, Heller SR, Montori VM, Seaquist ER, Service FJ. Evaluation and management of adult hypoglycemic disorders: An Endocrine Society Clinical Practice Guideline. J Clin Endocrinol Metab. 2009;94(3):709-728.

10. Druce MR, Muthuppalaniappan VM, O’Leary B, Chew SL, Drake WM, Monson JP, Akker SA, Besser M, Sahdev A, Rockall A, Vyas S. Diagnosis and localisation of insulinoma: the value of modern magnetic resonance imaging in conjunction with calcium stimulation catheterisation.Eur J Endocrinol. 2010;162(5):971-978. 
Bangladesh Journal of Medical Science Vol. 20 No. 01 January’21 